\title{
A DECOMPOSITION THEOREM FOR HOMOGENEOUS ALGEBRAS
}

\author{
L. G. SWEET and J. A. MACDOUGALL
}

Dedicated to Professor D. Z. Djoković

(Received 7 July 1999; revised 2 February 2001)

Communicated by B. A. Davey

\begin{abstract}
An algebra $A$ is homogeneous if the automorphism group of $A$ acts transitively on the one dimensional subspaces of $A$. Suppose $A$ is a homogeneous algebra over an infinite field $k$. Let $L_{a}$ denote left multiplication by any nonzero element $a \in A$. Several results are proved concerning the structure of $A$ in terms of $L_{a}$. In particular, it is shown that $A$ decomposes as the direct $\operatorname{sum} A=\operatorname{ker} L_{a} \oplus \operatorname{Im} L_{a}$. These results are then successfully applied to the problem of classifying the infinite homogeneous algebras of small dimension.
\end{abstract}

2000 Mathematics subject classification: primary 17D99; secondary 17A36.

Keywords and phrases: Non-associative algebras, automorphism group.

\section{Introduction}

The algebras to be discussed are assumed to be finite dimensional over a field $\mathbf{k}$ and are not necessarily associative. We call an algebra $A$ nontrivial if $\operatorname{dim} A>1$ and $A^{2} \neq 0$. Also, $\operatorname{Aut}(A)$ will denote the group of algebra automorphisms of $A$.

An algebra $A$ is homogeneous if $\operatorname{Aut}(A)$ acts transitively on the one-dimensional subspaces of $A$. This is a very strong condition indeed and the known examples fall into two easily described classes. The existence of homogeneous algebras depends critically on the choice of $\mathbf{k}$, the field of scalars, and a number of results are known classifying these algebras according to the field. Kostrikin [5] showed how to construct homogeneous algebras of any dimension over the finite field $\mathrm{GF}(2)$. Work by

(C) 2002 Australian Mathematical Society 0263-6115/2002 \$A2.00+0.00 
Shult [7], Gross [3] and Ivanov [4] showed that if $\mathbf{k}$ is finite, then there are no algebras other than those constructed by the method of Kostrikin. Djokovic [1] completely classified homogeneous algebras over the reals and found only 3 examples, one each in dimensions 3,6 and 7. It was shown by Sweet [10] that there are no non-trivial examples whatsoever when the scalar field is algebraically closed.

The first general study of homogeneous algebras was carried out by Sweet [9], and subsequently the authors $[6,8]$ have completely classified the non-trivial algebras of dimensions 2, 3 and 4 over any field. There it has been shown that no examples exist other than those found by Kostrikin and by Djoković. Recently, motivated by the examples over the reals, Djoković and Sweet [2] have shown that all non-trivial homogeneous algebras over any infinite field satisfy $x^{2}=0$ for all $x \in A$, and hence are anti-commutative.

The main purpose of this paper is to prove the following structure theorem which applies to homogeneous algebras over any infinite field. For any $a \in A, L_{a}: A \rightarrow A$ will denote left multiplication by $a$.

THEOREM. Let $A$ be a non-trivial homogeneous algebra over an infinite field. Then for any $a \in A \backslash\{0\}, A=\operatorname{ker} L_{a} \oplus \operatorname{Im} L_{a}$.

This theorem has a number of interesting consequences regarding the possible structure of infinite homogeneous algebras which will provide important tools in our continuing program of classifying these algebras.

In all that follows, $A$ will be a non-trivial homogeneous algebra over an arbitrary infinite field $\mathbf{k}$.

\section{Results and proofs}

One of the immediate consequences of homogeneity (see [9]) is that all left multiplications are projectively similar. More precisely, for any $a, b \in A$, if $\alpha \in \operatorname{Aut}(A)$ maps $a$ to $\lambda b$, then $\alpha L_{a} \alpha^{-1}=\lambda L_{b}$. This fact has been exploited very successfully in [6] and [8] to classify the homogeneous algebras of dimensions 2, 3 and 4. In particular we use the matrix representation of $L_{a}$ with respect to some suitably chosen basis. Note that if $a, b \in A \backslash\{0\}$ then $\operatorname{rank} L_{a}=\operatorname{rank} L_{b}$. Also if some coefficient of the characteristic polynomial of $L_{a}$ is zero then the corresponding coefficient of the characteristic polynomial of $L_{b}$ is also zero.

THEOREM 2.1. Let $A$ be a nontrivial homogeneous algebra over an infinite field $\mathbf{k}$. If $a \in A$, then $L_{a}$ has no nonzero eigenvalues in $\mathbf{k}$.

PROOF. In [2] it is proved that any homogeneous algebra $A$ over an infinite field 
has the property that $x^{2}=0$ for all $x \in A$. The theorem then follows from Theorem 3 of [8].

Our main result, which is Corollary 2.3, follows from the following theorem.

THEOREM 2.2. Let $A$ be a nontrivial homogeneous algebra over an infinite field $\mathbf{k}$. If $a, b \in A \backslash\{0\}$ and $a b=0$, then $\operatorname{Im} L_{a}=\operatorname{Im} L_{b}$.

PROOF. Choose a basis $B=\left\{b_{1}, b_{2}, \ldots b_{n}\right\}$ of $A$ such that $\left\{b_{1}, b_{2}, \ldots b_{s}\right\}$ is a basis of $\operatorname{ker} L_{a}$ and choose another basis $C=\left\{c_{1}, c_{2}, \ldots c_{n}\right\}$ such that $c_{i}=a b_{i}$ for $s<i \leq n$. Then the matrix of $L_{a}$ with respect to the bases $B$ and $C$ is

$$
\left[\begin{array}{cc}
O & O \\
O & I_{r}
\end{array}\right]
$$

where $r=n-s$ is the rank of $L_{a}$. Let $x \in A$ be arbitrary and let

$$
\left[\begin{array}{ll}
X_{1} & X_{2} \\
X_{3} & X_{4}
\end{array}\right]
$$

be the matrix of $L_{x}$ with respect to $B$ and $C$. Since the rank of $L_{x}+t L_{a}=L_{x+t a}$ cannot exceed $r$ for all $t \in k$, we conclude that $X_{1}=O$. Hence $x\left(\operatorname{ker} L_{a}\right)=$ $L_{x}\left(\operatorname{ker} L_{a}\right) \subset \operatorname{Im} L_{a}$. But $x$ is arbitrary and so $A\left(\operatorname{ker} L_{a}\right) \subset \operatorname{Im} L_{a}$. Since $b \in \operatorname{ker} L_{a}$, $A b=\operatorname{Im} L_{b} \subset \operatorname{Im} L_{a}$. But $\operatorname{rank} L_{a}=\operatorname{rank} L_{b}$ and so $\operatorname{Im} L_{a}$ and $\operatorname{Im} L_{b}$ have the same dimension. It follows that $\operatorname{Im} L_{a}=\operatorname{Im} L_{b}$.

COROLLARY 2.3. Let $A$ be a nontrivial homogeneous algebra over an infinite field k. If $a \in A \backslash\{0\}$, then $A=\operatorname{ker} L_{a} \oplus \operatorname{Im} L_{a}$.

PROOF. Let $b \in \operatorname{ker} L_{a} \cap \operatorname{Im} L_{a}$. If $b \neq 0$, then Theorem 2.2 implies that $\operatorname{Im} L_{a}=$ $\operatorname{Im} L_{b}$. But then $b \in \operatorname{Im} L_{b}$, which contradicts Theorem 2.1. Hence $\operatorname{ker} L_{a} \cap \operatorname{Im} L_{a}=$ $\{0\}$ and so $A=\operatorname{ker} L_{a} \oplus \operatorname{Im} L_{a}$.

Let $A$ be a nontrivial homogeneous algebra over a field $\mathbf{k}$ and let $a \in A$. If $\mathbf{k}$ is finite it was shown by Shult [7] that $L_{a}$ is either invertible or nilpotent. If $\mathbf{k}$ is infinite the first case is impossible since $a^{2}=0$. Also, if $\mathbf{k}$ is infinite the above corollary implies that the second case is also impossible. In fact, we have a slightly stronger result.

COROLLARY 2.4. Let A be a nontrivial homogeneous algebra over an infinite field k. If $a \in A \backslash\{0\}$, then $L_{a}$ cannot have a nonzero nilpotent block in its rational canonical form. 
Corollary 2.3 says that $A$ can be written as a direct sum of the subspaces $\operatorname{ker} L_{a}$ and $\operatorname{Im} L_{a}$. We now show that $\operatorname{ker} L_{a}$ is actually a subalgebra.

THEOREM 2.5. Let $A$ be a nontrivial homogeneous algebra over an infinite field $\mathbf{k}$. If $a \in A \backslash\{0\}$, then $\operatorname{ker} L_{a}$ is a zero subalgebra.

Proof. Assume $a \in A \backslash\{0\}$ and let $x \in A \backslash\{0\}$ be arbitrary. Using Corollary 2.3, decompose $A$ into $A=\operatorname{ker} L_{a} \oplus \operatorname{Im} L_{a}$. Then using a corresponding basis

$$
L_{a}=\left[\begin{array}{cc}
O & O \\
O & A_{1}
\end{array}\right] \text { and } L_{x}=\left[\begin{array}{ll}
X_{1} & X_{2} \\
X_{3} & X_{4}
\end{array}\right]
$$

Using the rank argument as in Theorem 2.2, we conclude that $X_{1}=O$ for all $x \in A$. Now let $b \in \operatorname{ker} L_{a} \backslash\{0\}$. Then

$$
L_{b}=\left[\begin{array}{cc}
O & B_{2} \\
B_{3} & B_{4}
\end{array}\right] .
$$

But $a b=0$ and so $\operatorname{Im} L_{a}=\operatorname{Im} L_{b}$ by Theorem 2.2. This implies that $B_{2}=O$. Also Corollary 2.3 implies that $A_{1}$ is nonsingular and so $B_{4}$ is also nonsingular since $L_{a}$ and $L_{b}$ are projectively similar.

Assume $B_{3} \neq O$. Then there exists $c \in \grave{\operatorname{ker}} L_{a}$ such that $b c=d \in \operatorname{Im} L_{a} \backslash\{0\}$. Since $B_{4}$ is nonsingular the equation $B_{4} x=d$ must have a unique solution $e \in \operatorname{Im} L_{a}$. But then $b c=b e$ and so $b(c-e)=0$. It follows that $\operatorname{Im} L_{b}=\operatorname{Im} L_{c-e}$. Since $c \in \operatorname{ker} L_{a}$, we again can assume that

$$
L_{c}=\left[\begin{array}{cc}
O & O \\
C_{1} & C_{2}
\end{array}\right]
$$

On the other hand,

$$
L_{e}=\left[\begin{array}{cc}
O & E_{2} \\
E_{3} & E_{4}
\end{array}\right] .
$$

But now $\operatorname{Im} L_{c-e}=\operatorname{Im}\left(L_{c}-L_{e}\right)=\operatorname{Im} L_{b}=\operatorname{Im} L_{a}$, and this implies that $E_{2}=0$. But $e \in \operatorname{Im} L_{a}$ and $e^{2}=0$ and so $E_{4}$ is singular. This is impossible since $L_{e}$ is projectively similar to $L_{a}$.

Hence $B_{3}=O$ and therefore $\operatorname{ker} L_{a}$ is a zero subalgebra.

COROLLARY 2.6. Let A be a nontrivial homogeneous algebra over an infinite field $\mathbf{k}$. If $a, b \in A \backslash\{0\}$ and $a b=0$, then $\operatorname{ker} L_{a}=\operatorname{ker} L_{b}$. Also, denoting $\operatorname{ker} L_{a} \backslash\{0\}$ by $K_{a}^{*}$, the sets $K_{a}^{\star}$ partition $A \backslash\{0\}$.

Proof. Assume $x \in \operatorname{ker} L_{b}$. Since $a \in \operatorname{ker} L_{b}$, Theorem 2.5 implies that $a x=0$, and so $x \in \operatorname{ker} L_{a}$. Hence $\operatorname{ker} L_{b} \subset \operatorname{ker} L_{a}$ and similarly $\operatorname{ker} L_{a} \subset \operatorname{ker} L_{b}$. Hence $\operatorname{ker} L_{a}=\operatorname{ker} L_{b}$. The proof of the second conclusion is similar. 
Theorem 2.5 shows that each $\operatorname{ker} L_{a}$ is a subalgebra. We now show that each $\operatorname{Im} L_{a}$ is not a subalgebra.

THEOREM 2.7. Let $A$ be a nontrivial homogeneous algebra over an infinite field $\mathbf{k}$. Let $a, b \in A \backslash\{0\}$. If $\operatorname{Im} L_{a}=\operatorname{Im} L_{b}$, then $\operatorname{ker} L_{a}=\operatorname{ker} L_{b}$.

ProOF. By using an argument similar to that found in Theorem 2.5 it can be shown that $a b=0$ and then the result follows directly from Corollary 2.6.

COROLLARY 2.8. Let $A$ be a nontrivial homogeneous algebra over an infinite field $\mathbf{k}$. If $a \in A \backslash\{0\}$, then $\operatorname{Im} L_{a}$ is not a subalgebra.

PRoof. Assume $\operatorname{Im} L_{a}$ is a subalgebra. Let $A=\operatorname{ker} L_{a} \oplus \operatorname{Im} L_{a}$. Suppose $b \in$ $\operatorname{Im} L_{a} \backslash\{0\}$. Then as before

$$
L_{b}=\left[\begin{array}{cc}
O & O \\
B_{3} & B_{4}
\end{array}\right]
$$

This implies that $\operatorname{Im} L_{a}=\operatorname{Im} L_{b}$ and so $\operatorname{ker} L_{b}=\operatorname{ker} L_{a}$. Thus $b \in \operatorname{ker} L_{a} \cap \operatorname{Im} L_{a}$ which is impossible.

It is natural to look at the action of an automorphism on $\operatorname{ker} L_{a}$ and $\operatorname{Im} L_{a}$. The next result is well known and the proof is easy.

REMARK. Let $A$ be any algebra over a field k. If $a \in A \backslash\{0\}, \alpha \in \operatorname{Aut}(A)$ and $\alpha(a)=b$, then $\alpha\left(\operatorname{ker} L_{a}\right)=\operatorname{ker} L_{b}$ and $\alpha\left(\operatorname{Im} L_{a}\right)=\operatorname{Im} L_{b}$.

COROLLARY 2.9. Let $A$ be a nontrivial homogeneous algebra over an infinite field $\mathbf{k}$. If $\alpha \in \operatorname{Aut}(A), a \in A \backslash\{0\}$ and $\alpha\left(\operatorname{ker} L_{a}\right) \cap \operatorname{ker} L_{a} \neq 0$, then $\alpha\left(\operatorname{ker} L_{a}\right)=\operatorname{ker} L_{a}$ and $\alpha\left(\operatorname{Im} L_{a}\right)=\operatorname{Im} L_{a}$.

PROOF. The proof follows easily from the above theorem using Corollary 2.6 and Theorem 2.5.

We now show that $\operatorname{Aut}(A)$ cannot be abelian if $A$ is a nontrivial homogeneous algebra over an infinite field $\mathbf{k}$ (the result is false when $\mathbf{k}$ is finite). Let $Z(\operatorname{Aut}(A))$ denote the center of $\operatorname{Aut}(A)$.

THEOREM 2.10. Let $A$ be a nontrivial homogeneous algebra over an infinite field $\mathbf{k}$. If $\alpha \in Z(\operatorname{Aut}(A))$ and $a \in A \backslash\{0\}$, then $\alpha\left(\operatorname{ker} L_{a}\right)=\operatorname{ker} L_{a}$ and $\alpha\left(\operatorname{Im} L_{a}\right)=\operatorname{Im} L_{a}$. 
ProOF. Let $\alpha \in Z(\operatorname{Aut}(A))$. We define a new multiplication $a \circ b$ on $A$ to make a new algebra $A^{\alpha}$ as follows:

$$
a \circ b=a \alpha(b)
$$

Then if $\gamma \in \operatorname{Aut}(A)$

$$
\gamma(a \circ b)=\gamma(a \alpha(b))=\gamma(a) \gamma(\alpha(b))=\gamma(a) \alpha(\gamma(b))=\gamma(a) \circ \gamma(b)
$$

and so $\gamma \in \operatorname{Aut}\left(A^{\alpha}\right)$. Thus $A^{\alpha}$ is a homogeneous algebra. Hence

$$
a \circ a=a \alpha(a)=0 .
$$

Thus $\alpha(a) \in \operatorname{ker} L_{a}, \forall a \in A$, and the result follows from Corollary 2.9.

COROLlARY 2.11. Let $A$ be a nontrivial homogeneous algebra over an infinite field $\mathbf{k}$. Then $\operatorname{Aut}(A)$ is not abelian.

PROOF. This follows immediately from Theorem 2.10.

The remaining theorems use the direct sum decomposition to study the possible dimension of $\operatorname{ker} L_{a}$.

THEOREM 2.12. Let $A$ be a nontrivial homogeneous algebra over an infinite field $\mathbf{k}$. If $a \in A \backslash\{0\}$, then $\operatorname{dim}\left(\operatorname{ker} L_{a}\right)<(1 / 2) \operatorname{dim}(A)$.

PROOF. Let $t=\operatorname{dim}\left(\operatorname{ker} L_{a}\right)$ and $n=\operatorname{dim}(A)$. Assume $t \geq n / 2$. Let $\left\{a_{1}, a_{2}, \ldots, a_{t}\right\}$ be a basis of $\operatorname{ker} L_{a}$ and decompose $a$ as $A=\operatorname{ker} L_{a} \oplus \operatorname{Im} L_{a}$. It follows from Theorem 2.5 that each $L_{a_{i}}$ is of the form

$$
L_{a_{i}}=\left[\begin{array}{cc}
O & 0 \\
O & A_{i}
\end{array}\right] .
$$

where $A_{i}$ is a nonsingular $(n-t) \times(n-t)$ matrix.

Since $t \geq n / 2$ there exists a nontrivial $b=x_{1} a_{1}+x_{2} a_{2}+\cdots+x_{t} a_{t}$ such that

$$
L_{b}=\left[\begin{array}{c|cccc}
O & & & 0 & \\
\hline & \star & \ldots & & \star \\
& \star & \ldots & & \star \\
O & \vdots & & & \vdots \\
& \star & \ldots & & \star \\
& 0 & \ldots & 0 & b_{n n}
\end{array}\right] .
$$

But then $b_{n n}=0$ by Theorem 2.1, and hence $L_{b}$ is not projectively similar to $L_{a}$. This is a contradiction and hence $t<n / 2$. 
THEOREM 2.13. Let $A$ be a nontrivial homogeneous algebra over an infinite field $\mathbf{k}$. If $n=\operatorname{dim} A$ is odd and $n>3$, then for $a \in A \backslash\{0\}, \operatorname{dim}\left(\operatorname{ker} L_{a}\right)<(n-1) / 2$.

PROOF. By Theorem 2.12 we know that $t=\operatorname{dim}\left(\operatorname{ker} L_{a}\right)<n / 2$ and so it suffices to show that $t \neq(n-1) / 2$. Assume otherwise. Decompose $A$ as $A=\operatorname{ker} L_{a} \oplus \operatorname{Im} L_{a}$. Let $b \in \operatorname{Im} L_{a}$ and let $B=\left\{b, b_{2}, \ldots, b_{t}\right\}$ be a basis for $\operatorname{ker} L_{b}$. Each $b_{i}$ can be written uniquely as

$$
b_{i}=a_{i}+b_{i}^{\prime}
$$

where $a_{i} \in \operatorname{ker} L_{a}$ and $b_{i}^{\prime} \in \operatorname{Im} L_{a}$. Let $B^{\prime}=\left\{b, b_{2}^{\prime}, \ldots, b_{t}^{\prime}\right\}$. We claim that $B^{\prime}$ is an independent set. For suppose $\lambda_{1} b+\lambda_{2} b_{2}^{\prime}+\ldots+\lambda_{t} b_{t}^{\prime}=0$. Then

$$
a\left(\lambda_{1} b+\lambda_{2} b_{2}^{\prime}+\cdots+\lambda_{t} b_{t}^{\prime}\right)=a\left(\lambda_{1} b+\lambda_{2} b_{2}+\cdots+\lambda_{t} b_{t}\right)=0 .
$$

So $\lambda_{1} b+\lambda_{2} b_{2}+\cdots+\lambda_{t} b_{t} \in \operatorname{ker} L_{a} \cap \operatorname{ker} L_{b}=\{0\}$. But $B$ is an independent set, and so $B^{\prime}$ must also be an independent set.

Let $c$ be any vector in the complement of the span of $B^{\prime}$ in $\operatorname{Im} L_{a}$. Then $B^{\prime} \cup\{c\}$ is a basis of $\operatorname{Im} L_{a}$. Now $b_{i} b_{i}^{\prime}=b_{i} a_{i} \in \operatorname{Im} L_{a}$ and so using any basis for $\operatorname{ker} L_{a}$ and $B^{\prime} \cup\{c\}$ as a basis of $\operatorname{Im} L_{a}$, we have

$$
L_{b_{i}}=\left[\begin{array}{c|ccccc} 
& 0 & 0 & \ldots & 0 & -b_{i 1} \\
& 0 & 0 & \ldots & 0 & b_{i 2} \\
O & \vdots & \vdots & & \vdots & \vdots \\
& 0 & 0 & \ldots & 0 & b_{i t} \\
\hline \star & & \star &
\end{array}\right] \text { and } L_{c}=\left[\begin{array}{c|c}
O & C_{1} \\
\hline C_{2} & \star
\end{array}\right] .
$$

Since $c \notin \operatorname{ker} L_{a}$, the columns of $C_{2}$ are independent and so rank $C_{2}=t$. Also $c \notin \operatorname{ker} L_{a}$ implies that $C_{1} \neq 0$ and so rank $C_{1}=1$. Since $n>3$ this implies that there exists a nonzero $b^{\prime}$ in the span of $B^{\prime}$ such that

$$
L_{b^{\prime}}=\left[\begin{array}{c|c}
O & O \\
\hline \star & \star
\end{array}\right]
$$

Since $b^{\prime} \notin \operatorname{ker} L_{a}$ this is impossible.

Our final result involves a lower bound for $\operatorname{dim}\left(\operatorname{ker} L_{a}\right)$. First we need the wellknown result described in the following lemma.

LEMMA 2.14. Let $M$ be an $n \times n$ matrix with entries from a field $\mathbf{k}$. Suppose $M$ is skew-symmetric and $m_{i i}=0$ for $1 \leq i \leq n$. If $n$ is odd, then $M$ is singular. 
THEOREM 2.15. Let A be a nontrivial homogeneous algebra over an infinite field $\mathbf{k}$. Let $a \in A \backslash\{0\}$. If $\operatorname{dim} A$ is even, then $\operatorname{dim}\left(\operatorname{ker} L_{a}\right)>1$.

Proof. Suppose $\operatorname{dim} A=n$ and let $a \in A \backslash\{0\}$. Then decompose $A$ as

$$
A=\operatorname{ker} L_{a} \oplus \operatorname{Im} L_{a} .
$$

Let $\left\{a, e_{2}, \ldots, e_{n}\right\}$ be the corresponding basis and assume $\operatorname{dim}\left(\operatorname{ker} L_{a}\right)=1$. We consider the top rows of $L_{e_{2}}, L_{e_{3}}, \ldots, L_{e_{n}}$. Since $A$ is anticommutative these rows are of the form

$$
\begin{array}{cc|cccccc}
R_{2}: & 0 & 0 & e_{23} & e_{24} & e_{25} & \ldots & e_{2 n} \\
R_{3}: & 0 & -e_{23} & 0 & e_{34} & e_{35} & \ldots & e_{3 n} \\
R_{4}: & 0 & -e_{24} & e_{34} & 0 & e_{45} & \ldots & e_{4 n} \\
\vdots & \vdots & & & & & & \\
R_{n}: & 0 & -e_{2 n} & -e_{3 n} & -e_{4 n} & -e_{5 n} & \ldots & 0
\end{array}
$$

Consider $x_{2} R_{2}+x_{3} R_{3}+\cdots+x_{n} R_{n}=0$. This is a homogeneous linear system of the form $M x=0$, where $M$ is a $(n-1) \times(n-1)$ skew-symmetric matrix, with $m_{i i}=0$ (we discard the first column). By Lemma 2.14, $M$ is singular and so the system has nontrivial solutions. Thus there exists a nonzero $x \in \operatorname{Im} L_{a}$ such that

$$
L_{x}=\left[\begin{array}{c|ccc}
0 & 0 & \cdots & 0 \\
\hline \star & & \star
\end{array}\right] .
$$

Again this is impossible since $x \notin \operatorname{ker} L_{a}$. This completes the proof.

\section{Homogeneous algebras of small dimension}

The general results described in the previous section are strong enough to limit the possible existence of homogeneous algebras having small dimension. Their real strength lies in the fact that they do not depend on the choice of the scalar field. These theorems allow us to dramatically shorten the work involved in classifying dimensions 2, 3 and 4, (as reported in [6] and [8]) and to make some additional useful observations.

We first briefly describe the only known examples of infinite homogeneous algebras. These exist over the real field and are described by Djoković in [1]. In that paper, he shows that there are only 3 such algebras. The first two are well-known: the 3dimensional algebra consisting of the pure quaternions and the 7-dimensional algebra consisting of the pure octonions. In both cases the multiplication is redefined to make $x^{2}=0$. There is also a 6-dimensional algebra $T=\mathbb{C}^{3}$ considered as a real vector space with multiplication as follows: for $x=\left(x_{1}, x_{2}, x_{3}\right)$ and $y=\left(y_{1}, y_{2}, y_{3}\right)$, let

$$
x \cdot y=\left(\overline{x_{2} y_{3}-x_{3} y_{2}}, \overline{x_{3} y_{1}-x_{1} y_{3}}, \overline{x_{1} y_{2}-x_{2} y_{1}}\right) \text {. }
$$


We conclude with a summary of the results of applying Theorems 2.12, 2.13 and 2.15 to algebras of dimension up to 7 . It may be that any further progress on classification will depend on specifying the scalar field $\mathbf{k}$.

- Dimension 2 By Theorem $2.15, \operatorname{dim}\left(\operatorname{ker} L_{a}\right)=2=\operatorname{dim}(A)$. Thus there are no non-trivial homogeneous algebras over any infinite field. This result was first shown in [9].

- Dimension 3 By Theorem 2.12, we must have $\operatorname{dim}\left(\operatorname{ker} L_{a}\right)=1$. Such an algebra exists as described above (also over certain other fields; see [6].

- Dimension 4 By Theorem 2.12, $\operatorname{dim}\left(\operatorname{ker} L_{a}\right)<2$, but by Theorem 2.15, $\operatorname{dim}\left(\operatorname{ker} L_{a}\right)>1$. Therefore there are no non-trivial homogeneous algebras over any infinite field. This is an improvement on the authors' work in [8].

- Dimension 5 According to Theorem 2.13, the only possibility for a homogeneous algebra is to have $\operatorname{dim}\left(\operatorname{ker} L_{a}\right)=1$. This case has not yet been resolved, but we conjecture no such algebra exists over any infinite field.

- Dimension 6 According to Theorems 2.12 and 2.15, the only possibility is for $\operatorname{dim}\left(\operatorname{ker} L_{a}\right)=2$. Such an algebra does exist over the reals, as described above.

- Dimension 7 By Theorem 2.13, there are two possibilities: $\operatorname{dim}\left(\operatorname{ker} L_{a}\right)=1$ or 2. The case of $\operatorname{dim}\left(\operatorname{ker} L_{a}\right)=1$ can occur: the algebra of pure octonions described above. The case of $\operatorname{dim}\left(\operatorname{ker} L_{a}\right)=2$ is unresolved, but we again conjecture no such algebra exists over any infinite field.

\section{Acknowledgement}

The authors would like to thank the anonymous referee for suggestions which improved both the content and presentation of this paper.

\section{References}

[1] D. Ž. Djoković, 'Real homogeneous algebras', Proc. Amer. Math. Soc. 41 (1973), 457-462.

[2] D. Ž. Djoković and L. G. Sweet, 'Infinite homogeneous algebras are anti-commutative', Proc. Amer. Math. Soc., 127 (1999) 3169-3174.

[3] F. Gross, 'Finite automorphic algebras over GF(2)', Proc. Amer. Math. Soc. 31 (1971), 10-14.

[4] D. N. Ivanov, 'On homogeneous algebras over $G F(2)$ ', Vestnik Moskov. Univ. Matematika 37 (1982), 69-72.

[5] A. I. Kostrikin, 'On homogeneous algebras', Izvestiya Akad. Nauk USSR 29 (1965), 471-484.

[6] J. A. MacDougall and L. G. Sweet, 'Three dimensional homogeneous algebras', Pacific J. Math. 74 (1978), 153-162.

[7] E. E. Shult, 'On the triviality of finite automorphic algebras', Illinois J. Math. 13 (1969), 654-659.

[8] L. G. Sweet and J. A. MacDougall, 'Four dimensional homogeneous algebras' Pacific J. Math. 129 (1987), 375-383. 
[9] L. G. Sweet, 'On homogeneous algebras', Pacific J. Math. 59 (1975), 385-594.

[10] , 'On the triviality of homogeneous algebras over an algebraically closed field', Proc. Amer. Math. Soc. 48 (1975), 321-324.

Department of Mathematics and Computer Science University of Prince Edward Island Charlottetown PEI C1A 4P3 Canada e-mail: sweet@upei.ca
Department of Mathematics University of Newcastle Callaghan NSW 2308

Australia e-mail: mmjam@cc.newcastle.edu.au 